\title{
BMJ Open Identifying the facilitators, constraints and barriers of community indoor walking programmes: protocol for a realist synthesis
}

\author{
Shaminder Singh (D) , ${ }^{1}$ Lin Yang, ${ }^{2,3}$ Sonia Butalia, ${ }^{1,4}$ Hude Quan, ${ }^{1}$ \\ Tanvir C Turin (D) 1,5
}

To cite: Singh S, Yang L, Butalia S, et al. Identifying the facilitators, constraints and barriers of community indoor walking programmes: protocol for a realist synthesis. BMJ Open 2020;10:e034342. doi:10.1136/ bmjopen-2019-034342

- Prepublication history for this paper is available online. To view these files, please visit the journal online (http://dx.doi org/10.1136/bmjopen-2019034342).

Received 16 September 2019 Revised 21 May 2020 Accepted 25 June 2020

Check for updates

(C) Author(s) (or their employer(s)) 2020. Re-use permitted under CC BY-NC. No commercial re-use. See rights and permissions. Published by BMJ.

For numbered affiliations see end of article.

Correspondence to

Dr Shaminder Singh;

shasingh@ucalgary.ca

\section{ABSTRACT}

Introduction Physical inactivity is a costly and leading health risk factor. Engaging in moderate or more intense regular physical activity reduces premature mortality at the population level. Walking is a viable option for achieving the recommended level of physical activity. Yet, the sedentary lifestyle is trending. Determinants of physical activity may be personal, social or environmental. Health promotion endeavours aiming to enhance population-level physical activity are reported in the literature. However, a full range of factors influencing the development and implementation of sustainable indoor walking programmes is unclear. The current review protocol is aimed at describing a process of realist synthesis to uncover contexts, mechanisms and outcomes of indoor walking intervention programmes, which might reveal facilitators, constraints and barriers of planning, implementing and participating in indoor walking initiatives open for the members of the general public.

Methods and analysis We will employ a realist synthesis to determine successes or failures in certain circumstances for specific stakeholders, which will aid in developing a sustainable mall walking health promotion and community engagement programme. Qualitative, quantitative and mixed-method articles and reports will be screened for intervention theories and models in order to identify elements of programmes that may be linked to the success or failure of the interventions. Data related to the context, mechanism and outcome of the interventions will be collected, analysed and synthesised iteratively until a theoretical understanding develops, which might explain the intricacies of the success and failure of identified indoor walking programmes. The review process will be conducted and evaluated by using the recommended tools.

Ethics and dissemination Ethical approval, such as Conjoint Health Research Ethics Board, was not required for this study because no direct interaction with patients will occur for data collection and analysis. We will disseminate directly to the scholarly community through publication and presentation and may post on social media or websites.

PROSPERO registration number CRD42020150415.
Strengths and limitations of this study

- In addition to reporting the outcomes, the realist synthesis will explore contexts and mechanisms to review the success and failures of the programmes.

- A wide range of expertise of our transdisciplinary team might be helpful in developing an inclusive initial programme theory, thereby reducing selection bias.

- The explorative and iterative approach of the discovery might reveal relevant components of indoor programmes and what drives the successes and failures for various stakeholders.

- This protocol lacks a step-by-step review procedure because the realist synthesis is inherently an iterative and interactive process.

- English language, rehabilitation and treatmentbased exclusions might omit components of community indoor walking programmes from the review that may be relevant for the members of the general public.

\section{BACKGROUND}

Physical inactivity is a foremost risk factor of chronic illnesses (such as vascular diseases and cancer) ${ }^{1}$ and mortality, ${ }^{2}$ which costs an estimated 2013 Int' $1 \$ 53 \cdot 8$ billion globally. ${ }^{3}$ Engaging in regular moderate physical activity (such as a 20 min daily brisk walking) can reduce the risk of premature mortality by $16 \%-30 \%$ at the population level. ${ }^{4}$ However, a sedentary lifestyle is trending globally ${ }^{5}$ despite its established links with health risks, ${ }^{16}$ public awareness ${ }^{7}$ and accessibility of low-cost physical activity such as walking.

Physical activity may be determined by any combinations of personal (eg, awareness, perception, health status), social (eg, context, accessibility, services) or environmental (eg, safety, transportation connectivity-walking, bicycling or public transit to programme destinations, destination proximity) characteristics or features. ${ }^{7-13}$ 
A majority of respondents in a Canadian survey $(67 \%$; $\mathrm{n}=2519$ ) attributed physical inactivity to both personal and public health responsibilities. ${ }^{7}$ Community walking programmes may enhance physical activity at the population level. ${ }^{14}{ }^{15}$ Walking requires no specific skills or equipment and can be an accessible way of engaging in physical activity for its health benefits. ${ }^{16}$ In a literature review, Hanson and Jones ${ }^{17}$ located 42 studies reporting outdoor walking programmes from 14 countries (mostly in the USA; $n=15$ ) and attended by 19 and older individuals $(n=1843)$ who yielded improved health outcomes with no adverse effects of attending. Walking has shown the population-level physical and mental health benefits including a reduction of depressive symptoms, cardiovascular risk factors and all-cause mortality. ${ }^{18-21}$

Farren $e t a l^{22}$ found that people engage in indoor physical activities such as mall walking programmes for their indoor climate-controlled, safe and comfortable environments. In places like Canada, where harsh winter weather conditions can limit outdoor activities, low or no-cost indoor walking programmes at publicly accessible spaces can be a feasible option to increase physical activity among people at the population level. Local stakeholders' engagement in programme development and implementation, such as involving mall managers and members of catchment communities, may enhance the adoption of indoor walking initiatives.

We aim to develop a model that may help local communities to plan and implement sustainable indoor walking programmes open for the members of the general public. However, we are unaware of what enables, initiates or maintains (facilitators) indoor community walking programmes, what discourages or blocks (barriers) and what limits the scope or sustainability (constraints) of such initiations. For example, a shared interest between an indoor facility manager and the catchment community may facilitate the initiation of an indoor walking programme, but a potential burden of insurance liability may discourage the launch of the programme posing as a barrier. The scope of an ongoing programme may face constraints such as limited public transportation. A systematic search and screening ${ }^{23}$ of the existing literature of indoor community walking programmes and its realist synthesis ${ }^{24}$ may offer an opportunity to expose the links between contexts and processes of programme development, implementation and evaluation. We plan to use the knowledge created from this proposed study to engage various community stakeholders for designing indoor community walking programmes by enhancing facilitators, negotiating constraints and addressing potential barriers.

\section{Objectives}

The initial review questions are:

1. How well realist evaluation components are used, if any, to report indoor community walking programmes?
Keywords for programs:

(interven* or program ${ }^{*}$ or promo* or initiat* $^{*}$ or implement ${ }^{\star}$ or group $^{*}$ or communit* or indoor or campaign ${ }^{\star}$ or impact* or project* or servic $^{\star}$ or pattern $\left.{ }^{\star}\right)$.tw,kf.

\section{Keywords for Indoor Walking:}

(walk* and (indoor or inside or built* or interior ${ }^{*}$ or mall* or hall ${ }^{\star}$ or stair $^{*}$ or atrium ${ }^{*}$ or atria* or theatre ${ }^{*}$ or theater ${ }^{*}$ or seminary or building ${ }^{*}$ or facilities or facility or center ${ }^{*}$ or centre* or institute $^{*}$ or school* or university* or college ${ }^{*}$ or campus ${ }^{*}$ or church ${ }^{*}$ or synagogue ${ }^{*}$ or temple ${ }^{*}$ or mosque* or gurudwara* or gurdwara* or place ${ }^{*}$ or area* or office* or workplace* or "work place ${ }^{\star *}$ or "at work" or site* or space* or spot*)).tw,kf.

Figure 1 Systematic search strategy. The figure illustrates a systematic strategy to search records in the literature containing organised indoor community walking group programmes.

2. What are the facilitators, constraints and barriers of indoor community walking programmes for participants as well as organisers and service providers?

3. What characteristics of an indoor community walking programme make it both successful and unsuccessful in enhancing physical activity?

4. For whom do indoor community walking programmes work or not work, to what extent and in what ways?

5 . What are the processes of programme development, promotion and participation as well as mechanisms of successful implementation and participation of indoor community walking, and in what way they are meaningful for future initiatives?

\section{METHOD}

In order to select search terms, the review questions were divided into two categories ${ }^{23}:$ (1) community programmes and (2) indoor walking. Figure 1 contains a detailed list of the two categories of search terms. A community walking programme is defined as an organised walking group available for all people at minimal or no cost to the participants. We will include indoor community walking group programmes open for all people to attend, which are designed to increase the physical activity of members of the general public and that may offer insights into the facilitators, constraints or barriers of joining, sustaining, conducting or maintaining the programmes. A group programme must contain two or more regular members walking at a relatively consistent frequency, time and location. We will exclude non-English articles and walking programmes that were (1) explicitly designed for people with specific illnesses or health conditions such as aimed at rehabilitation, (2) conducted as a one-time or an occasional walking event and (3) combined with other structured physical activities such as breathing patterns, Yoga or Tai Chi. However, non-physical activities, such as information sessions or interventions related to diet and nutrition, will not be excluded.

The search sources will include academic databases and grey literature with no restriction to a time frame. Table 1 consists of a list of both sources. Specialised databases will be searched to identify relevant articles based on predetermined search terms presented in figure 1. Grey literature 


\begin{tabular}{|c|c|}
\hline Academic databases: & Grey literature: \\
\hline MEDLINE (Ovid) & Google \\
\hline EMBASE (Ovid) & Google Scholar \\
\hline PsycINFO & $\begin{array}{l}\text { ProQuest (theses and } \\
\text { dissertations) }\end{array}$ \\
\hline Scopus & $\begin{array}{l}\text { Canadian Institute for Health } \\
\text { Information }\end{array}$ \\
\hline Web of Science & Public Health Agency of Canada \\
\hline CINAHL & Health Canada \\
\hline SOcINDEX & National Institutes of Health \\
\hline Urban Studies Abstracts & Canadian Electronic Library \\
\hline SPORTDiscus & \\
\hline
\end{tabular}

is especially important for the review because the government and non-government organisations which develop, modify and use community walking programmes may not necessarily publish their reports in specific databases. Snowballing (citation tracking) and purposive sampling (to find answers to specific questions) strategies will be used to search relevant programme theories (set of assumptions that highlight features of intervention) in the grey literature. A backward and forward reference list of identified articles will be searched. The protocol is registered with PROSPERO. Any significant amendment in the proposed protocol will be documented in the registered PROSPERO record.

\section{Identification and screening process of the studies}

The identified articles through the comprehensive search will be collected in a bibliography database, and the duplicates will be removed by using Covidence systematic review software, which compares and removes identical articles based on embedded metadata of the items. The inclusion and exclusion criteria are described in box 1 .

The collected articles will be further selected for review based on the inclusion and exclusion criteria. The

\section{Box 1 Inclusion and exclusion criteria for searched}

\section{articles}

Inclusion criteria (must meet all):

1. A walking programme organised in an indoor public space.

2. Indicated at least one barrier, facilitator, constraint to attend, sustain, conduct or maintain the walking programme.

3. Open for the members of the general public.

4. Any study design.

Exclusion criteria (at least meet one):

1. A one-time or a seasonal walking event.

2. Designed for a population with specific illnesses, disabilities or health conditions.

3. Combined with other structured physical activities such as Yoga, Tai Chi or breathing patterns.

4. Non-English articles.

5. Non-primary research articles.

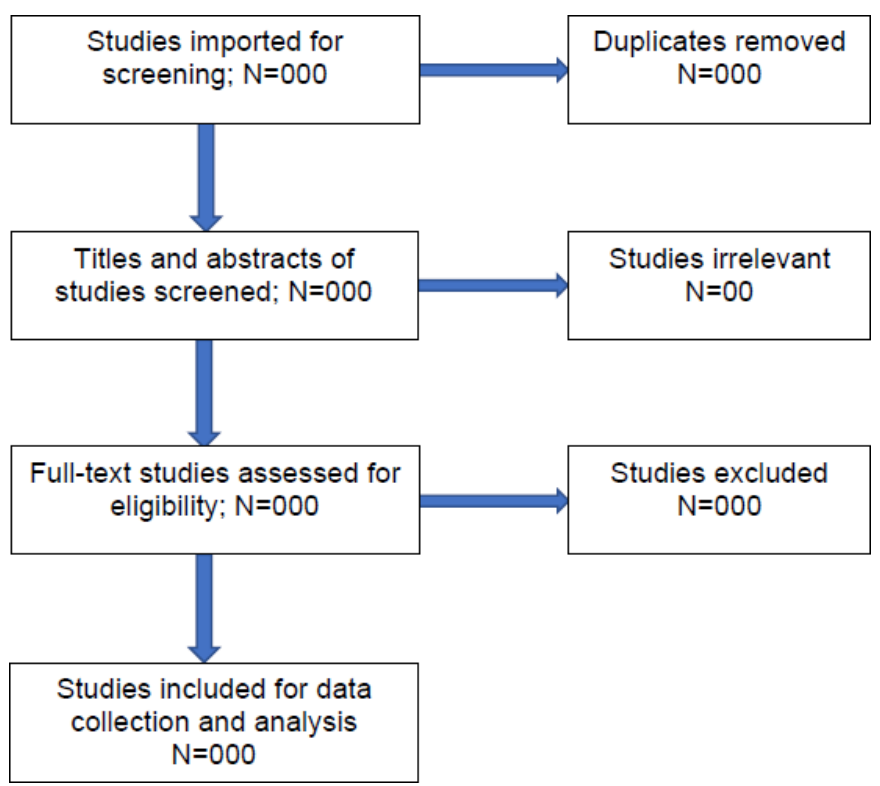

Figure 2 Identification and screening process of the studies based on the Preferred Reporting Items for Systematic Reviews and Meta-Analyses flow diagram. The figure illustrates a systematic process to identify and remove duplicate records searched from the literature, scrutinise records based on inclusion and exclusion criteria and, finally, select the relevant records for the review.

selection procedure will consist of two related steps: (1) identification by title and abstract screening and (2) full text for screening. The titles and abstracts reveal key information about a research article to identify its relevance for the review. Articles will be selected based on titles and abstracts, and a selection consensus will be made between two independent reviewers. In the case of a disagreement between the reviewers, similar to Brown et $a l^{25}$ a third reviewer will resolve any discrepancies. Articles will be excluded on the agreement of at least two of the independent reviewers. Both the article identification and screening process are based on the Preferred Reporting Items for Systematic Reviews and Meta-Analyses flow diagram, ${ }^{26}$ which is illustrated in figure 2.

Reviewers will read and tabulate data from the finally selected articles in table 2. Data will be extracted related to the study characteristics (year of publication, country) as well as the facilitators, constraints and barriers of indoor walking programmes. A multitude of qualitative methods can be used to explore the programme development processes and experiences of a mall walking intervention. The reviewers will extract the information related to methods used in the articles, the number of participants and their gender, recruitment strategies used (frequency and duration), the type of walking group (eg, mall or school) and its duration and other qualitative aspects such as location and transportation provided or arranged by the participants, incentives offered and use of technology such as mobile app or accelerometers. Each reviewer will identify and list all the facilitators, constraints and barriers of attending, sustaining, conducting or maintaining the 


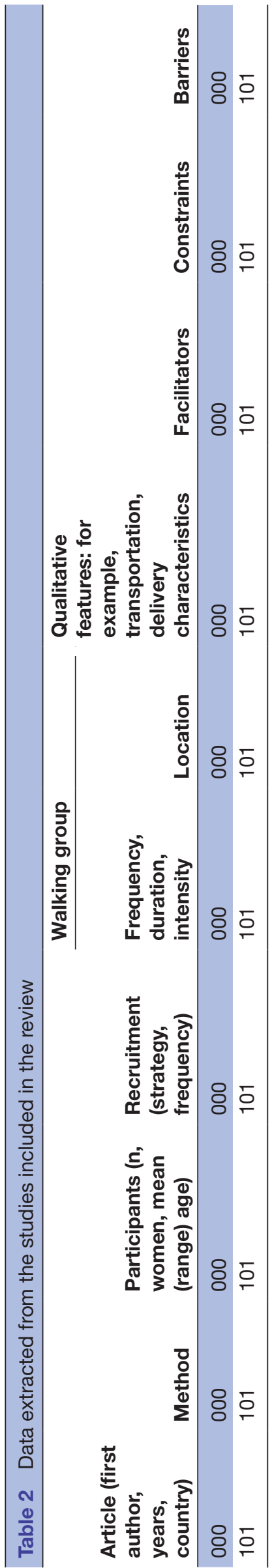

walking programmes. A consensus will be made among at least two of the reviewers in order to keep the inferences for the realist synthesis. The data will be recorded and saved in a Microsoft Excel sheet.

\section{Quality assessment}

All the included studies will be scrutinised for quality assessment. The quality of quantitative studies will be assessed using strategies similar to Brown et al. ${ }^{25}$ The first two authors will evaluate the quality of the intervention programmes using the Effective Public Health Practice Project Quality Assessment Tool for Quantitative Studies-a recommended tool to assess the content and construct validity of the studies. The quality assessment tool will be used to rate quantitative studies by a rating of weak, moderate or strong on six aspects: design, blinding, bias, confounders, methods of data collection and dropouts (researchers loose contact with participants) and withdrawals (participants state a reason for the discontinuation of their partaking). The aggregate assessment will be taken into account while reporting the quality of the selected studies. The results will be reported using table 3 .

The reviewers will assess the qualitative studies by the rigour and relevance of the included articles, which will be appraised based onRealist And MEta-narrative Evidence Syntheses: Evolving Standards ${ }^{24} 27$ The Cochrane Qualitative and Implementation Methods Group (CQIMG) ${ }^{28}$ recommended the reviewers' transparency in reporting the shared decision-making process of the quality assessment. We will assess and report the quality of the synthesised qualitative studies using table 4 , which is based on Critical Appraisal Skills Programme (CASP) ${ }^{29}$ CASP is the most frequently used tool to synthesise qualitative evidence in the WHO and Cochrane guidelines. ${ }^{28} \mathrm{We}$ will use the CASP as a guiding tool to assess and not as a scoring checklist because the score-based consensus is impractical while synthesising qualitative research. In doing so, the included articles may consist of a variety of methods with incomparable theoretical and philosophical frameworks. ${ }^{28}$ Instead of the cut-off points, the CQIMG ${ }^{28}$ recommended two or more reviewers to develop an understanding of the strengths and limitations of the included studies using guidelines such as the CASP.

\section{Realist synthesis}

Health promotion community interventions uniquely interplay with a context, which may not invoke similar dynamics when replicated in a different community and at another place and time. Therefore, a synthesis of such knowledge requires a perspective to appreciate the contextuality of an intervention that can respond to the question of 'What works for whom under what circumstances, how and why?'. ${ }^{24}$ We plan to use realist synthesis suggested by Pawson $e t a l^{4}$ for the review. Previously, other researchers applied realist synthesis to explore physical activity promotion strategies. ${ }^{30} 31$

Pawson $e t a l^{24}$ proposed that rather than being a method, the realist synthesis offers a 'logic of inquiry' to explore 
Table 3 Results of the quality assessment tool

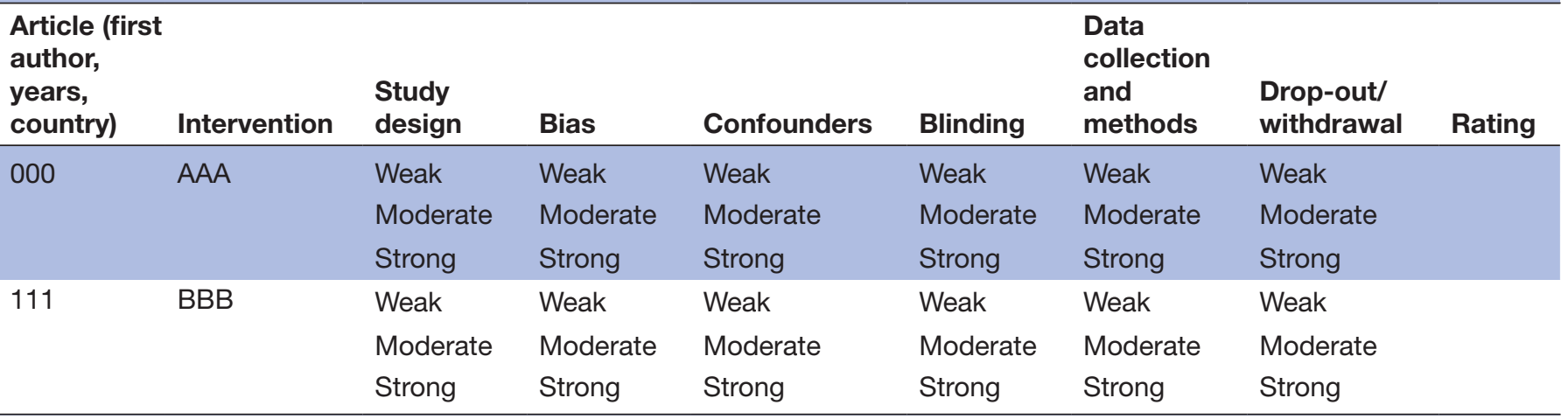

a phenomenon of interest. The review process includes hypothesising and testing the mechanics, context and the outcomes of social interventions. ${ }^{24}$ Realist synthesis examines the successes and failures of the interventions as well as inquiring about the processes and their respective contexts, including the salient features of the programmes and the social reality surrounding it. Realist synthesis uses all methods of inquiry, such as qualitative, quantitative or mixed methods.

The search process, identification, screening and analysis are parallel and iterative processes in the logic of realist synthesis. ${ }^{24}$ The review process begins with a background literature search and identification of existing programme theories, which exposes its features such as administrative ideologies, places, environments and social interactions. Low or no-cost publicly available health promotion (including non-rehabilitative) indoor walking programmes may exist widely, but they are rarely theorised from the perspective of programme development, implementation, participation and sustainability. We conducted an environmental scan in Calgary (Alberta, Canada) and found several (un)supervised walking groups available for the members of the general public that run for years without being theorised. Currently, we are conducting a preliminary literature search to develop an initial programme theory for publicly accessible mall walking programmes that are unrestricted based on people's age, gender, ethnicity, diseases or disability.

The initial programme theory will inform further literature search in a focused manner based on the features of the identified indoor walking programmes. Further, the primary research questions may be modified as the new knowledge uncovers about the phenomenon in the literature. Snowballing and purposive sampling will aid in finding answers to the analytic questions that may emerge from the ongoing search of the identified programme theories, which may further mobilise the review and synthesise process in order to resolve unanswered questions. Components of the programme theories will be searched, identified and tested through the literature search, which may modify the research question, data analysis and synthesis, making it an interactive and iterative process.

Pawson $e t a t^{24}$ argued that realist synthesis is a 'refining theory', which is achieved through parallel processes of data collection and analysis. Data will be extracted by tracking the components of the programme theories and analysis will be carried out by constant comparing between what works in what circumstances and what are the conditions it did not work. ${ }^{24}$ Reviewers will strive to locate the programme ideologies and frameworks as well as implementation processes, including evolution in the

Table 4 Critical Appraisal Skills Programme qualitative research checklist

Questions $^{29}$

Yes Cannot tell No

Was there a clear statement of the aims of the research?

Is a qualitative methodology appropriate?

Was the research design appropriate to address the aims of the research?

Was the recruitment strategy appropriate to the aims of the research?

Was the data collected in a way that addressed the research issue?

Has the relationship between researcher and participants been adequately considered?

Have ethical issues been taken into consideration?

Was the data analysis sufficiently rigorous?

Is there a clear statement of findings?

How valuable is the research? 
intervention strategies. With a new understanding of programme intervention, the reviewers will raise questions and find their answers in the empirical literature to develop a better understanding of what works (or not) and in what circumstances.

\section{Patient and public involvement}

No patient involved in this study. Patient consent was not required for data collection, analysis or publication of this study.

\section{DISCUSSION}

This article is an account of our reflection on the process of a realist synthesis that we plan to undertake in order to understand what works (or not) for an indoor walking programme and discovering the challenges, for whom, and in what circumstances. We hope that the resulting realist synthesis of literature will provide a comprehensive understanding of the potential facilitators, constraints and barriers existing in the literature. This knowledge will be shared with local community liaisons and stakeholder consultations, which will be instrumental in planning and implementing a walking programme for health promotion and disease risk reduction.

The expected outcome of this review is to yield a better understanding of what elements of indoor walking programmes were successfully adopted by whom and in which circumstances. The resulting knowledge will help the reviewers to design an indoor community walking programme by selecting a potentially appropriate setting and using intervention components that will be deemed to have an optimum positive influence on populationwide participation and behaviour change.

The traditional models of analysis and synthesis of intervention programmes primarily rely on clinical trials, which are aimed to understand the causal relationship of an event or an outcome. However, such traditional reviews tend to omit contextual knowledge important to community-based or public health interventions because clinical trials focus merely on outcomes and strive to control extraneous variables that cannot be removed in real-life interventions. Realist synthesis, in addition to outcomes, facilitates to explore the underlying mechanisms of complex intervention programmes by taking its context into account. ${ }^{24} 32$ The proposed realist synthesis may reveal what influences health promotion efforts related to indoor walking programmes in both urban and rural settings.

The planned realist synthesis will be a critical step in the process of reducing the prevalence of physical inactivity at the population level. The review is expected to reveal components of an indoor walking programme that may be suitable for various groups of individuals such as mall managers and the members of the catchment community. The resulting knowledge might help indoor space managers to develop accessible and sustainable indoor walking programmes combining its components.
The proposed review is aimed at synthesising knowledge for its application into the real world by developing and implementing a community indoor walking programme, which is essentially a knowledge translation endeavour as we will use research evidence into practice. This review may ultimately inform broader public health dissemination and implementation research related to indoor walking programmes.

The walking programme will be appraised by a realist evaluation in order to refine the programme further, and it might be a stepping stone for an ongoing community engagement aiming to reduce population physical inactivity.

\section{Limitations}

The iterative realist synthesis may not provide definitive answers to the research questions. ${ }^{24}$ The realist synthesis is not designed to report the success of the intervention programmes based on the outcomes only. Instead, it will review the contexts and mechanisms leading to the outcomes, which will be instrumental in developing an informed framework of intervention.

The initial programme theory development is underway, which will further determine the search and review directions. The systematic search explained in this review will guide the development of the initial programme theory, which may introduce a selection bias as we will likely select the features of the model based on the research team's experiences and expertise. However, our transdisciplinary research team brings a wide variety of expertise, which might aid in developing an inclusive initial programme theory.

Another limitation of this protocol is the absence of a step-by-step review procedure. The realist synthesis is inherently open ended and iterative and subjected to take turns and twists as the synthesis matures, so it is not feasible to delineate the review process precisely and in advance. Instead, this protocol is a guiding tool to initiate the process, to keep the aim of the synthesis into focus as the review progresses and to establish the transparency of the review and synthesis processes.

\section{Ethics and dissemination}

Recruitment or direct interactions of patients or members of the general public will not take place in the proposed study. Ethical approval, such as Conjoint Health Research Ethics Board, was not required for the study. The process of the realist synthesis will be reported transparently for critics, researchers and implementation specialists.

The facilitators, constraints and barriers uncovered from the study will inform the development of a community indoor walking health promotion programme. The findings of the study will be shared with the academic community through peer-reviewed publications, conferences presentations or posters, webinars and formal and informal meetings. We will disseminate the findings to the community through the programme website, social media and conventional media such as radio and use it 
to communicate with stakeholders while developing the indoor walking programme.

\section{Registration}

The study protocol is registered with PROSPERO. Currently, we are conducting a preliminary literature search that will help us develop an initial programme theory. We anticipate completing the study by October 2020.

\section{Author affiliations \\ ${ }^{1}$ Community Health Sciences, Cumming School of Medicine, University of Calgary, Calgary, Alberta, Canada \\ ${ }^{2}$ Cancer Epidemiology and Prevention Research, Alberta Health Services, Calgary, Alberta, Canada \\ ${ }^{3}$ Department of Oncology, Cumming School of Medicine, University of Calgary, Calgary, Alberta, Canada \\ ${ }^{4}$ Department of Medicine, Cumming School of Medicine, University of Calgary, Calgary, Alberta, Canada \\ ${ }^{5}$ Department of Family Medicine, Cumming School of Medicine, University of Calgary, Calgary, Alberta, Canada}

\section{Twitter Sonia Butalia @Sonia_ButaliaMD}

Collaborators Vince Avati; Camilia Thieba; Katelyn Corkin.

Contributors SS, TCT, LY, SB and HQ conceptualised the work and critiqued the study protocol. SS and TCT designed the protocol and drafted the initial version of the manuscript as well as developed data extraction and appraisal strategy and the tables. The study problem, objectives and the search terms were defined in team meetings where SS, TCT, LY, SB and HQ directly contributed. Similarly, the whole team defined and refined the inclusion and exclusion criteria and contributed to the manuscript with critical revisions of the initial draft.

Funding This work was supported by Astra Zeneca Cardiovascular Health Promotion and Disease Prevention Chair awarded to Dr Hude Quan.

Competing interests None declared.

Patient consent for publication Not required.

Provenance and peer review Not commissioned; externally peer reviewed.

Open access This is an open access article distributed in accordance with the Creative Commons Attribution Non Commercial (CC BY-NC 4.0) license, which permits others to distribute, remix, adapt, build upon this work non-commercially, and license their derivative works on different terms, provided the original work is properly cited, appropriate credit is given, any changes made indicated, and the use is non-commercial. See: http://creativecommons.org/licenses/by-nc/4.0/.

\section{ORCID iDs}

Shaminder Singh http://orcid.org/0000-0002-5076-0863

Tanvir C Turin http://orcid.org/0000-0002-7499-5050

\section{REFERENCES}

1 Warburton DER, Bredin SSD. Health benefits of physical activity: a systematic review of current systematic reviews. Curr Opin Cardiol 2017;32:541-56.

2 World Health Organization. Global health risks: mortality and burden of disease attributable to selected major risks 2009.. Available: http:// www.who.int/healthinfo/global_burden_disease/global_health_risks/ en/

3 Ding D, Lawson KD, Kolbe-Alexander TL, et al. The economic burden of physical inactivity: a global analysis of major non-communicable diseases. Lancet 2016;388:1311-24.

4 Ekelund U, Ward HA, Norat T, et al. Physical activity and allcause mortality across levels of overall and abdominal adiposity in European men and women: the European prospective investigation into cancer and nutrition study (EPIC). Am J Clin Nutr 2015;101:613-21.

5 Ng SW, Popkin BM. Time use and physical activity: a shift away from movement across the globe. Obes Rev 2012;13:659-80.

6 Tremblay MS, Warburton DER, Janssen I, et al. New Canadian physical activity guidelines. Appl Physiol Nutr Metab 2011;36:47-58.
7 Yun L, Vanderloo L, Berry TR, et al. Assessing the social climate of physical (in)activity in Canada. BMC Public Health 2018;18:1301.

8 Yang L, Sahlqvist S, McMinn A, et al. Interventions to promote cycling: systematic review. BMJ 2010;341:c5293.

9 O'Donoghue G, Kennedy A, Puggina A, et al. Socio-economic determinants of physical activity across the life course: a "DEterminants of Dlet and Physical ACtivity" (DEDIPAC) umbrella literature review. PLoS One 2018;13:e0190737.

10 Condello G, Puggina A, Aleksovska K, et al. Behavioral determinants of physical activity across the life course: a "DEterminants of Dlet and Physical ACtivity" (DEDIPAC) umbrella systematic literature review. Int J Behav Nutr Phys Act 2017;14:58.

11 Cortis C, Puggina A, Pesce C, et al. Psychological determinants of physical activity across the life course: A "DEterminants of Dlet and Physical ACtivity" (DEDIPAC) umbrella systematic literature review. PLoS One 2017;12:e0182709.

12 Farkas B, Wagner DJ, Nettel-Aguirre A, et al. Evidence synthesis - A systematized literature review on the associations between neighbourhood built characteristics and walking among Canadian adults. Health Promot Chronic Dis Prev Can 2019;39:1-14.

13 Nichani V, Vena JE, Friedenreich CM, et al. A population-based study of the associations between neighbourhood walkability and different types of physical activity in Canadian men and women. Prev Med 2019:129:105864.

14 South J, Giuntoli G, Kinsella K, et al. Walking, connecting and befriending: a qualitative pilot study of participation in a lay-led walking group intervention. J Transp Health 2017;5:16-26.

15 U.S. Department of Health and Human Services. Step it up! the surgeon general's call to action to promote walking and walkable communities. Washington DC: U.S, 2015.

16 Ogilvie D, Foster CE, Rothnie $\mathrm{H}$, et al. Interventions to promote walking: systematic review. BMJ 2007;334:1204.

17 Hanson S, Jones A. Is there evidence that walking groups have health benefits? a systematic review and meta-analysis. $\mathrm{Br} J$ Sports Med 2015;49:710-5.

18 Hamer M, Chida Y. Walking and primary prevention: a meta-analysis of prospective cohort studies. Br J Sports Med 2008;42:238-43.

19 Robertson R, Robertson A, Jepson R, et al. Walking for depression or depressive symptoms: a systematic review and meta-analysis. Ment Health Phys Act 2012;5:66-75.

20 Kelly P, Kahlmeier S, Götschi T, et al. Systematic review and metaanalysis of reduction in all-cause mortality from walking and cycling and shape of dose response relationship. Int J Behav Nutr Phys Act 2014;11:132.

21 Murtagh EM, Nichols L, Mohammed MA, et al. The effect of walking on risk factors for cardiovascular disease: an updated systematic review and meta-analysis of randomised control trials. Prev Med 2015;72:34-43.

22 Farren L, Belza B, Allen P, et al. Mall walking program environments, features, and participants: a scoping review. Prev Chronic Dis 2015;12:E129.

23 Ahmed S, Vaska M, Turin TC. Comprehensive systematic search process of health literature: hunting pearls out of the sea. J Natl Heart Found Bangladesh 2006:12-16.

24 Pawson R, Greenhalgh T, Harvey G, et al. Realist review--a new method of systematic review designed for complex policy interventions. J Health Serv Res Policy 2005;10 Suppl 1:21-34.

25 Brown HE, Atkin AJ, Panter J, et al. Family-based interventions to increase physical activity in children: a meta-analysis and realist synthesis protocol. BMJ Open 2014;4:e005439.

26 Moher D, Liberati A, Tetzlaff J, et al. Preferred reporting items for systematic reviews and meta-analyses: the PRISMA statement. PLoS Med 2009;6:e1000097.

27 Wong G, Greenhalgh T, Westhorp G, et al. RAMESES publication standards: realist syntheses. BMC Med 2013;11:21.

28 Noyes J, Booth A, Flemming K, et al. Cochrane qualitative and implementation methods group guidance series-paper 3: methods for assessing methodological limitations, data extraction and synthesis, and confidence in synthesized qualitative findings. J Clin Epidemiol 2018;97:49-58.

29 Critical Appraisal Skills Programme. CASP qualitative checklist 2018 Available: https://casp-uk.net/wp-content/uploads/2018/01/CASPQualitative-Checklist-2018.pdf [Accessed 28 Aug 2019].

30 Leone L, Pesce C. From delivery to adoption of physical activity guidelines: realist synthesis. Int J Environ Res Public Health 2017;14:ijerph14101193.

31 Brown HE, Atkin AJ, Panter J, et al. Family-based interventions to increase physical activity in children: a systematic review, metaanalysis and realist synthesis. Obes Rev 2016;17:345-60.

32 Pawson R. Evidence-based policy. London, UK: SAGE, 2006. 